\title{
Design and Simulation of a Service Robot for Covid-19 Isolation Wards with Autonomous Navigation
}

\author{
Lalit Duseja", Yash Deshmukh, Shantanu Karmuse, Dr. S. S. Ohol \\ Department of Mechanical Engineering, College of Engineering, Pune, Maharashtra, India
}

(Received 31 August 2021; Accepted 10 October 2021)

DOI: https://doi.org/10.36224/ijes.140302

\begin{abstract}
The robots that are used to carry out the daily repetitive tasks in any type of environments are service robots. These robots have a great potential to eliminate human intervention and reduce contact in order to decrease the spread of virus. Service robots are of great importance when the human society is hit with a situation of pandemic as they can help in serving the patients in and infectious environment and isolating them from the surroundings. Such kind of a service robot that would serve infected patients in isolation wards is designed \& simulated by taking in the consideration the requirements. The dimensions of robot such as to optimize workspace requirements and the simulation of position and task of supplying the deliverables to patients from buffer area to isolation area are presented. The designed robot can successfully deliver medicines, food etc.to the patients without any contact between doctors, nurses or patients by using an on boarded Radio Frequency Identification system. The robot is well equipped with the sanitization mechanism to disinfect itself.
\end{abstract}

Keywords: Covid-19, Service Robot, Optimized Design, Autonomous Robot, Dijkstra's algorithm, MATLAB, RFID

\section{Introduction}

In recent days, a great amount of effort is being put in the development of nurse robots in order to serve the infected patients with dangerous viruses in an infectious environment and better products are introduced in the markets. Carnegie Mellon University along with University of Michigan developed a robot that assists in the nursing homes and is of a great help to the old and elderly people. It provides services to them such as reminding them of their daily tasks to take medicines, help them exercise etc. Care-O-Bot-II [2] developed by Germany can help elderly people to live independently, to perform placing table and chair, etc. This robot can autonomously navigate and find its route in indoor environments [3]. VA-PAMAID [4] from Eire, can help senior citizens or people suffering from as the nopia to detect obstacles and walk independently. Integrating the advanced electronics with service robots can help to make them more safe and reduce human intervention. Also, on boarding them with IOT can make them more interactive. A robot that would work autonomously in isolation wards and deliver the items to patients safely will be presented in this paper. This robot would help the doctors, nurses, relatives of patients to provide the patients with food, medicines and other deliverables by eliminating the contact between infected and non-infected people.

\section{Literature Review}

After the first wave of Covid-19, many service robots were introduced to tackle the situation. Coro bot from PNT solutions is a sanitizing service robot and can be operated through an app. Another robot that has he ability to navigate inside the isolation wards on its own is KARMI BOT. PARO, a therapeutic 
robot, has following sensors: tactile, audition, light, temperature, and posture sensors, using which it can perceive people and its environment. Apart from these robots, service robots like TUG Robot, Robear, Pepper robot are being developed and used over the past few years.

\section{Problem definition}

This study aims to develop a robot that delivers the belongings like food and medicine from the acquaintances of the patients and the medics to the patients within an isolation ward. The robot should be able to pick up multiple boxes at once from a pickup station and place them on the tables alongside the patients' beds and return the empty boxes to the pickup station. This design comprises 3 basic mechanisms that include the delivery mechanism, the chassis of the mobile robot, and the sanitization mechanism to disinfect the boxes after each delivery. Design of crucial components such as bearings and shafts of wheels, links of mechanisms, mobile chassis has to be done using traditional machine techniques and should be verified using FEM procedures. Dijkstra's algorithm is implemented for optimal path planning which would help in reducing the delivery time and hence ensuring the freshness of edible items. For the sake of convenience of the acquaintances, they will be provided with an RFID card, unique for each patient to instruct it to deliver as well as to bring back the boxes.

\section{Method of analysis}

\subsection{Design of Box Delivery Mechanism}

The slider-crank mechanism is used to transfer the boxes from robot to patient. The possibility of sag in the links is prevented by providing support to the weight of the box using linear rail carriages. Worm geared DC motor is used for lifting and lowering the box. The worm gear provides the self-locking ability which locks the position of the arm. A rope would be used to hold the carriages that support the arm to lift or lower the box. A spool would be coupled to the shaft around which the rope would be wound. Two DC motors are used, one actuates the crank and the other varies the angular position of the mechanism (refer Figure 1).

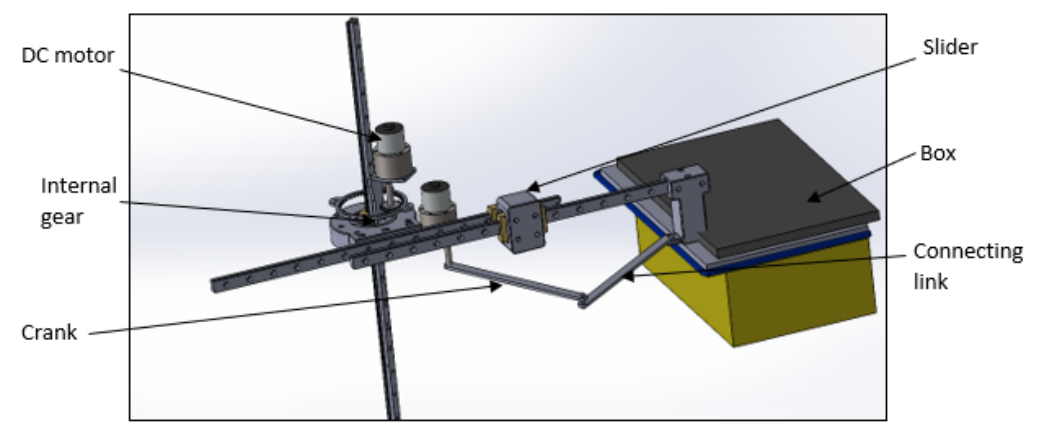

Figure 1: Slider crank mechanism

Considering the weight of the box to be $2 \mathrm{~kg}$ along with DC motors and slider, the rope pulls up this system, so it will be acted on by a force of $40 \mathrm{~N}$. For a motor with integrated worm gearbox having a rated torque of $16 \mathrm{Nm}$ and a factor of safety of 2, any diameter of the pulley less than $400 \mathrm{~mm}$ should work. To vary the radial and angular positions of the arm, Orange Johnson Geared DC Motor is used. The Orange Johnson geared motor is a rotary actuator with high speed and low torque, and has a variety of standard gear boxes available. It's programmable \&number of turns can be easily monitored when an encoder is coupled with a motor. It has input voltage range of 6-18 volts, a stall torque of $350 \mathrm{~N}-\mathrm{cm}$. The 
speed at rated voltage of $12 \mathrm{~V}$ is $200 \mathrm{rpm}$. For high-speed applications, a $1000 \mathrm{rpm}$ motor is also available.

Let $l 1=$ length of crank

$12=$ length of connecting rod, refer (Figure 2)

Max. Extension $=\frac{\text { Length of robot }}{2}-68.93 \mathrm{~mm}$

Max Extension $=l 1+l 2=\frac{827.7}{2}-68.93=344 \mathrm{~mm}$

As $l 2>=l 1$, for crank to rotate $360^{\circ}$

For maximum extension, $l 1-l 2=0$ or $l 1=l 2$

Substituting, lengths of the crank and the connecting link are:

$$
l 1=l 2=\frac{344}{2}=172 \mathrm{~mm}
$$

The maximum tensile stress encountered in the rail comes out to be 3.6 times less than the yield stress of structural steels, the design is safe.

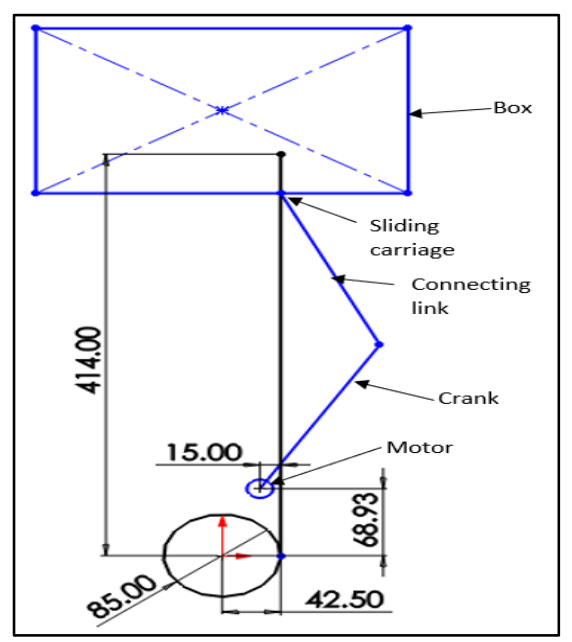

Figure 2: Layout of mechanism

\subsection{Design of Chassis for Mobile Robot}

Chassis helps the robot to locomote from one point to another point on the ground. The type of drive used is a 4-wheeled skid-steer drive for easy locomotion on smooth surfaces and simple automation. The mobile robot will have 4 wheels which will help it to locomote with ease. The motors used in the chassis are brushed DC motors with an external gear reduction of 52:1. Wheels of external diameter $127 \mathrm{~mm}$ are used to provide enough torque and to keep the CG of basedrive close to ground to avoid toppling. Mathematical modeling of four wheeled drive [5] helps in better control and motion on diverse terrain. $\left[T_{1}^{0}\right]=$

$$
\left[\begin{array}{cccc}
\cos (\mathrm{q} 1) & -\cos (\mathrm{q} 4 / 2) \sin (\mathrm{q} 1) & \sin \left(\frac{\mathrm{q} 4}{2}\right) \sin (\mathrm{q} 1) & \mathrm{q} 2 \cos (\mathrm{q} 1)-95 \cos (\mathrm{q} 4 / 2) \sin (\mathrm{q} 1)-175 \cos (\mathrm{q} 1)-\mathrm{q} 3 \sin (\mathrm{q} 1) \\
\sin (\mathrm{q} 1) & \cos (\mathrm{q} 4 / 2) \cos (\mathrm{q} 1) & -\sin (\mathrm{q} 4 / 2) \cos (\mathrm{q} 1) & 95 \cos (\mathrm{q} 4 / 2) \cos (\mathrm{q} 1)-175 \sin (\mathrm{q} 1)+\mathrm{q} 3 \cos (\mathrm{q} 1)+\mathrm{q} 2 \sin (\mathrm{q} 1) \\
0 & \sin (\mathrm{q} 4 / 2) & \cos (\mathrm{q} 4 / 2) & 95 \sin (\mathrm{q} 4 / 2) \\
0 & 0 & 0 & 1
\end{array}\right]
$$

$\mathrm{qi}=$ Transformation corresponding to shift in axes $\mathrm{x}, \mathrm{y}, \mathrm{z}$, or transformation corresponding to rotation around defined axes represents coordinate qi. 


\subsubsection{Speed of the robot}

DC motors are characterized with less torque and high speed. In order to attain the sufficient torque, a gear box has to be deployed with the motor to sustain the weight of the whole robot and provide a smooth motion to it. Since torque required to accelerate the robot from rest for $1 \mathrm{sec}$ to a velocity of 2 $\mathrm{m} / \mathrm{sec}$ happens to be $1.404 \mathrm{Nm}$, a gear reduction ratio of 52:1 is selected from the available standard choices as per the catalogue to operate the motor at half of its rated torque. $2.46 \mathrm{~m} / \mathrm{s}$ is the maximum speed that the robot would attain.

$$
\begin{gathered}
\text { Angular speed of motor }(\omega)=\frac{\text { No load angular speed }}{\text { Gear ratio }}(4) \\
\omega=19300 / 52=371 \mathrm{RPM}=38.86 \mathrm{rad} / \mathrm{s}(5) \\
\text { Velocity of wheel }(v)=\omega^{*} r=38.86^{*} 0.127 / 2=2.46 \mathrm{~m} / \mathrm{s}
\end{gathered}
$$

\subsubsection{Chassis}

As the chassis has a complex geometry, traditional approach can't be used. So, FEA tool is used to analyze the integrity of a hollow weldment of rectangular sections. From the force analysis, bearing of diameter of $15 \mathrm{~mm}$ is sufficient. SKF bearings with a designation of 6002 can be used.

The colored zones, refer(Figure 3a) represents the magnitudes of deformation of chassis. Since the load of the mechanism will be acting on chassis in vertical direction, it will deform. The maximum deformation is indicated by the red zone and its magnitude is $0.9 \mathrm{~mm}$. This is a small deformation and can be sustained by the aluminium chassis.

The colored zones, refer (Figure $3 b$ ) represent the von mises stress induced in the chassis due to load of mechanism. The blue zones indicate the area of minimum stress while red zone indicates maximum stress. The maximum stress induced in the chassis is $37.5 \mathrm{MPa}$ which is less than the yield strength of aluminium with an applied factor of safety. Hence the design of chassis is safe.

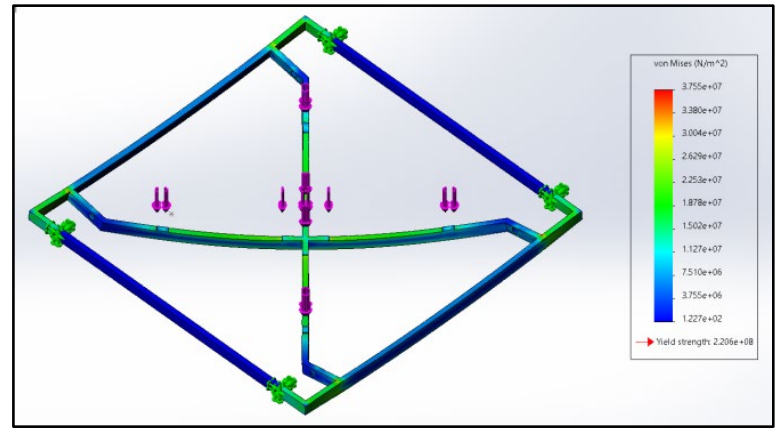

Figure 3 a: Deformation of chassis

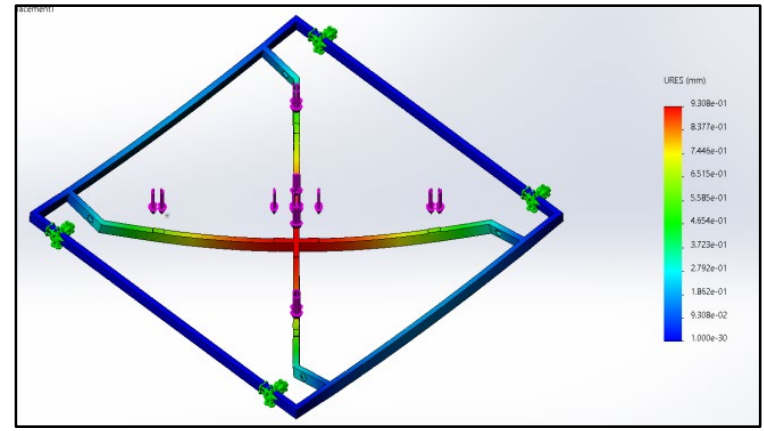

Figure 3 b: Stress distribution in chassis

\subsection{Sanitizing Mechanism}

To stop the spread of the virus the boxes need to be sanitized frequently after each use. To fulfill this requirement, the sanitizer is sprayed on the boxes with the help of a hose with multiple holes that is aligned along the edges of arm that picks and places the box refer (Figure 4b). The sanitizer is stored at the top of the robot in a closed container and is pumped in the sprayer using a 6V DC Pump with a lift of $0.4-1.1 \mathrm{~m}$ and flow rate of $80-120 \mathrm{~L} / \mathrm{H}$ 
4.4 .
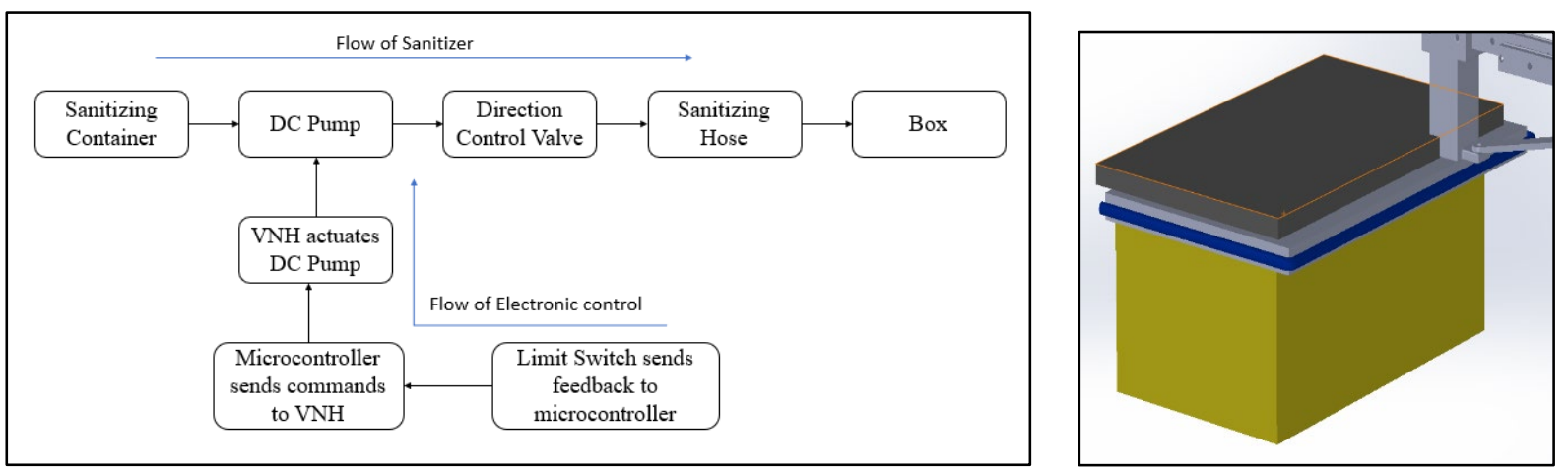

Figure 4 a: Block Diagram of sanitization mechanism

Figure 4 b: Sanitizing mechanism

Electronics and Instrumentation:

a. RFID: Radio-frequency identification (RFID) makes use of electromagnetic waves to scan and decipher the code on the RFID tag. Passive tags provided to the acquaintances are energized from the radio waves that are emitted by RFID reader (within the robot). This will ensure that the unique RFID cards given to the acquaintances of the patients can recognize the boxes coming from them and deliver those to the right patients.RFID technology use automatic data capture system which helps in increasing system efficiency[6].

b. Keyes KY-040 Arduino Rotary Encoder is used to measure the coordinates of the arm in the cylindrical coordinate system. The encoder would help to determine the angular, radial positions and elevation of the arm with respect to the base frame located on the chassis.

c. Autonics e $50 \mathrm{~s}$ is an incremental optical rotary encoder. It helps in the indoor navigation of the mobile robots. It is coupled with the wheel and counts the number of rotations of wheels. Using this count encoder can determine the distance travelled and provides it as a feedback. It has a high resolution of $500 \mathrm{~Hz}$.

d. LSA08 is a line following sensor that has 8 IR transmitter and receiver pairs. It is used by the robots to perform the task of line following. The floor of the wards would be mapped with black colored strips which would help robot to find its path from one point to other. The surrounding lights have minimal effect on the sensors readings.

e. Arduino nano: It is a compact and user-friendly microcontroller and has ATmega328 integrated in it . Integrated Development Environment (IDE) is used for programming the microcontrollers and one such IDE for Arduino nano is Arduino IDE.Arduino IDE consists of predefined libraries which helps in controlling the motors as per the needs of the terrain.

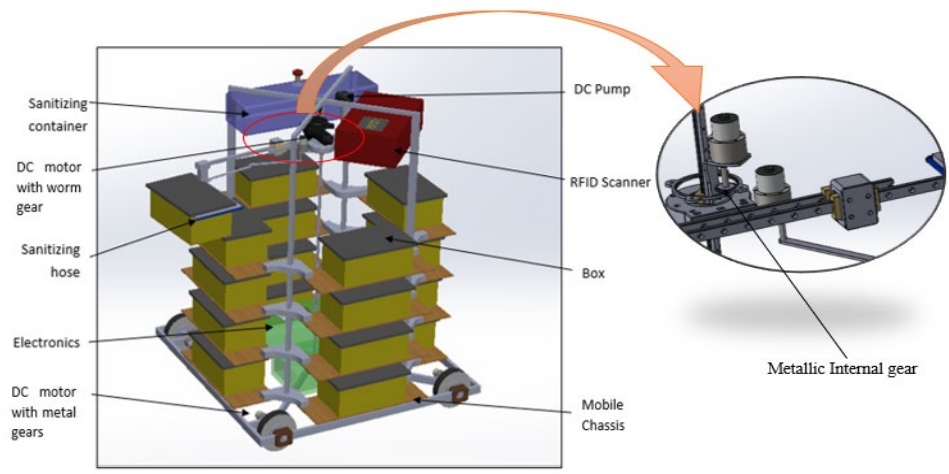

Figure 5: SolidWorks model of robot 
Table 1: Specifications of the robot

\begin{tabular}{|c|c|c|}
\hline Sr. No. & Parameter & Value \\
\hline $\mathbf{1 .}$ & Dimensions without extension & $830 \times 830 \times 1000 \mathrm{~mm}^{3}$ \\
\hline $\mathbf{2}$. & Dimensions with extension & $830 \times 1000 \times 1000 \mathrm{~mm}^{3}$ \\
\hline $\mathbf{3}$ & Mass of the robot & $43.2 \mathrm{~kg}$ \\
\hline $\mathbf{4}$ & Maximum linear speed & $2.46 \mathrm{~m} / \mathrm{s}$ \\
\hline $\mathbf{5 .}$ & Number of boxes on the robot & 16 \\
\hline $\mathbf{6}$. & Payload Capacity of each box & $2 \mathrm{~kg}$ \\
\hline $\mathbf{7 .}$ & Sanitizer tank capacity & $500 \mathrm{~mL}$ \\
\hline $\mathbf{8 .}$ & Number of batteries (Lithium Polymer) & 3 \\
\hline $\mathbf{9}$ & Charge capacity of each battery & $5200 \mathrm{mAh}$ \\
\hline $\mathbf{1 0}$ & Rated Voltage of each battery & $12 \mathrm{~V}$ \\
\hline $\mathbf{1 2}$ & Average current consumption & $20 \mathrm{Amp}$ \\
\hline $\mathbf{1 3}$ & Average Power consumption & $240 \mathrm{~W}$ \\
\hline $\mathbf{1 4}$ & Endurance of robot & $45 \mathrm{minutes}$ \\
\hline $\mathbf{1 5}$ & Approximate cost of prototyping & Rs. 57400 \\
\hline
\end{tabular}

\subsection{Development of Virtual Model in MATLAB}

Simscape, a feature of MATLAB, has the ability to create models of physical and real systems within the Simulink environment. The virtual model is developed using MATLAB. It is developed in three phases. First is the scripting phase. Dijkstra's algorithm solves the problem of finding the shortest path from a point in a graph (the source) to a destination[8]. Second phase is building the block diagram of Robot in Simulink and preparing the virtual environment for simulation. Third phase is the interfacing of script and the block diagram.

Dijkstra's algorithm is well developed and there are various models available for this. We have used the developed algorithm [9] and modified it to meet our needs. It is scripted in MATLAB. It takes the input of graph, the starting point and the destination point. In order to define the graph, we have to provide the two matrices. The nodal coordinate matrix and the nodal connectivity matrix defines the floor plan. The function returns the shortest distance and the shortest route that the robot must follow. The code is modified to take input of multiple destination points and then calculate which patient is closest from starting point and the shortest route. The robot should go to that patient following this route. Now the starting point should be reset and made equal to the destination point of patient 1 . The code should compute which is the next closest patient from this new starting point and the shortest route for the robot. This process continues until all the destinations are visited.

A $3 \mathrm{~d}$ model of robot and its environment is prepared using a $3 \mathrm{~d}$ modelling software which is then converted to .xml file. In order to simulate the results in MATLAB, this xml file is then imported by retaining all of the constraints. Importing $\mathrm{xml}$ file in MATLB can be done using the following command: smimport("file_name.xml"). The path of the xml file should be added in the MATLAB.

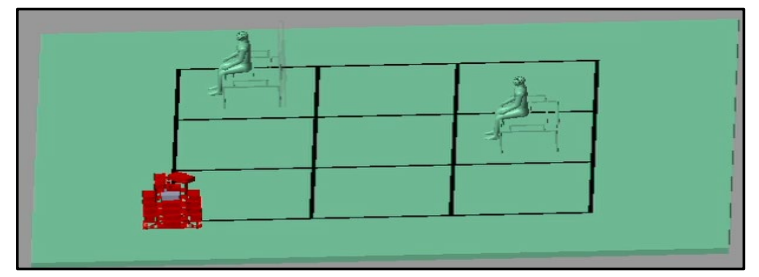


Figure 6: Mechanics Explorer view of robot in ward

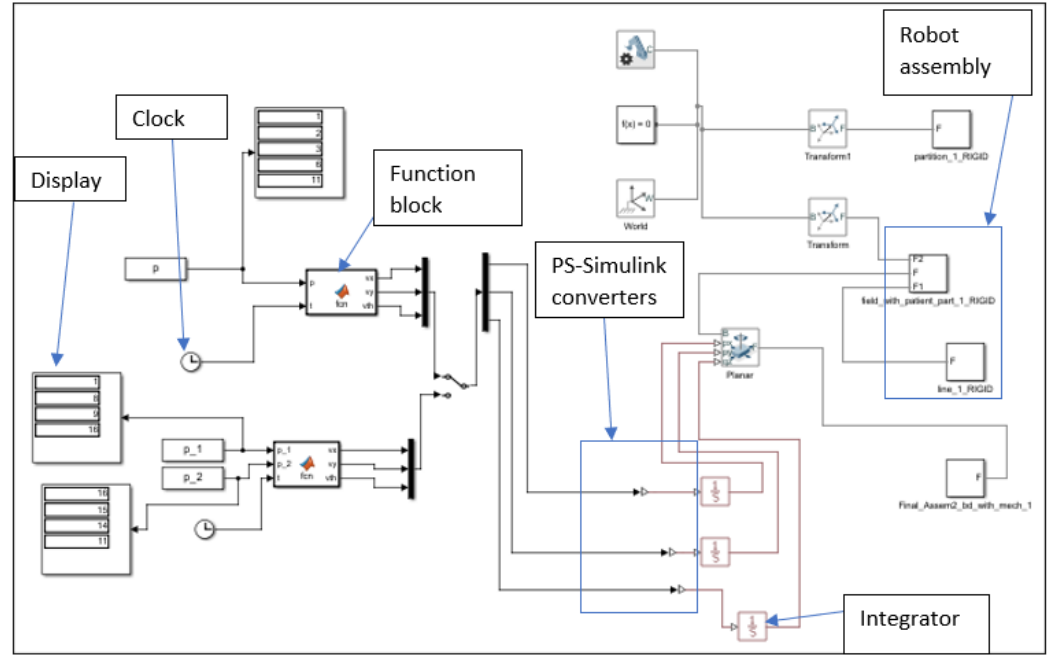

Figure 7: Block Diagram after interfacing

\section{Results and discussion}

The robot is designed in such a way that it can pass between the patients easily without causing trouble to the patients. The robot would move with its arms closed that is in the retracted position which makes its dimension $830 \times 830 \times 1000 \mathrm{~mm}^{3}$. A $6 \mathrm{~V} \mathrm{DC}$ pump would be deployed on the robot to pump the sanitizer fluid. The weight distribution of the robot is symmetrical and the elements such as electronics, sanitizer tank etc. are placed close to the center in order to coincide the center of mass with the geometric center of robot. This would reduce the chances of toppling of robot. Geared DC motors with a gear ratio of 52:1 are used to actuate the wheels of robot for easy and efficient operation. Dijkstra's algorithm successfully helps in minimizing distance of travel which helps in reducing the delivery time to ensure the freshness of food items and medicines.

\section{Conclusions}

This paper described the design of a service robot that would deliver the medicines and food to the patients of Covid-19 Isolation wards with the minimal contact. These kinds of service robots can be proven very useful during pandemic times to safeguard the doctors and nurses. Division of robot into multiple subsystems make the robot modular. The design of robot is in such a way that the chassis and the mechanism can be separated from each other which can be used for various other purposes as well. Implementing 4-wheeled skid steer drive would be cot effective and efficient for such kind of system and a maximum speed of $2.46 \mathrm{~m} / \mathrm{s}$ can be achieved. The virtual model helps in predicting the performance parameters of robot such as the delivery time which is obtained to be 32 seconds for delivering to two patients in a certain environment.

\section{References}

[1] Pineau J, Montemerlo M, Pollack M et al. Towards robotic assistants in nursing home: Challenges and results. Robotics and Autonomous Systems, 2003,42(3): 271-281

[2] Graf B, Hans M, Kubacki J et al. Robotic home assistant care-o-bot II. In: Proceedings of the 2002 IEEE EMBS/BMES Conference, Houston. 2002: 2343-2344. 
[3] Graf, Birgit \& Hans, Matthias \& Schraft, Rolf, Care-O-bot II-Development of a Next Generation Robotic Home Assistant, Autonomous Robots 16(2):193-205, 2004

[4] Lacey G, MacNamara S. User involvement in the design and evaluation of a smart mobility aid. Rehabilitation Resources Development, 2002,37(6):709_723

[5] Gracia, L., Tornero, J. Kinematic control of wheeled mobile robots. Latin American Applied Research,. Vol. 38, p. 7-16, 2008.

[6] Davinder Parkash, Twinkle Kundu, Preet Kaurthe, RFID Technology and its Applications: A Review. International Journal Of Electronics, Communication \& Instrumentation Engineering Research And Development (IJECIERD) ISSN 2249-684x Vol.2, Issue 3, Sep 2012, 109-120

[7] Oscar C, Yasuhisa H, Kazuhiro K. Control of walking support systems based on variable center of rotation. In: Proceeding of 2004 IEEE/RSJ International Conference on Intelligent Robots and Systems (IROS), New York. 2004: 2289-2294.

[8] Javaid, Adeel, Understanding Dijkstra Algorithm. SSRN Electronic Journal, 2013, 10.2139/ssrn.2340905.

[9] Dimas Aryo, Dijkstra Algorithm (https://www.mathworks.com/matlabcentral/fileexchange/36140-dijkstraalgorithm), 2021, MATLAB Central File Exchange. Retrieved June24, 2021. 